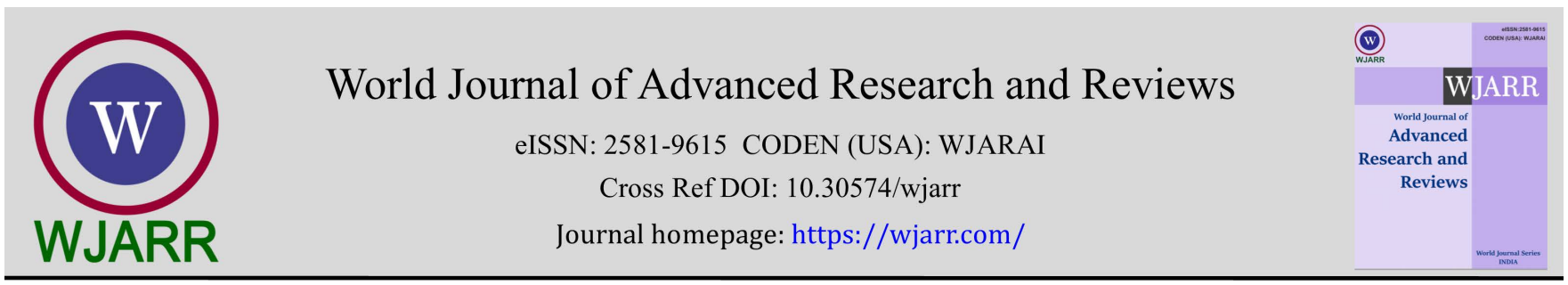

(RESEARCH ARTiClE)

\title{
Effect of Tai Chi Chuan practice on immune system and pro-metastasis markers in early post-treatment breast cancer survivors
}

\author{
Mary NB Cheung ${ }^{1}$ and Wings Tjing Yung Loo ${ }^{2, *}$ \\ ${ }^{1}$ Institute for Research and Practice of Yang Style Tai Chi Chuan, Hong Kong. \\ ${ }^{2}$ Keenlink Dental Clinic \& Essence Medical Laboratory, Hong Kong.
}

World Journal of Advanced Research and Reviews, 2021, 12(01), 013-019

Publication history: Received on 20 August 2021; revised on 28 September 2021; accepted on 30 September 2021

Article DOI: https://doi.org/10.30574/wjarr.2021.12.1.0472

\begin{abstract}
Purpose: To investigate the effect of Tai Chi Chuan (TCC) to improve immune system and decrease pro-metastasis markers in early post-treatment breast cancer survivors.

Methods: 130 post-treatment breast cancer survivors were recruited and randomized 1:1 into TCC group and wait-list (control) group. The TCC group practiced for a 60-minutes session once per week, for a total of 52 weeks. 115 forms of Yang-style TCC were taught by a Tai Chi master. Blood samples were taken from each subject and complete blood count was performed. The expressions of NKG2D protein, P-selectin, and vascular endothelial growth factor (VEGF) in plasma were measured. Lymphocyte activity was measured by cell proliferation reagent and ATP assay. Images of lymphocyte colony formation were taken with an inverted microscope.
\end{abstract}

Results: At 52 weeks, TCC group demonstrated a significantly higher WBC ( $p=0.001)$, a significantly higher NKG2D value ( $p=0.001)$ and a significantly lower VEGF value $(p=0.005)$ when compared to the wait-list group. However, there was a small, non-significant change for P-selectin values between the breast cancer survivor groups. After $72 \mathrm{~h}$ incubation, TCC group had a significant increase in lymphocyte proliferation $(p=0.001)$ and greater area of lymphocyte clusters or colonies $(p=0.001)$.

Conclusion: The practice of TCC could stimulate tumor immunosurveillance via NKG2D and activate the immune response. VEGF, a marker playing an important role in breast cancer and its metastases, was also reduced in those who practiced TCC. As an alternative for conventional exercise, post-treatment breast cancer survivors may select TCC in their rehabilitation program.

Keywords: Tai Chi Chuan; Breast cancer survivors; NKG2D; VEGF; Lymphocyte proliferation

\section{Introduction}

In 1985, the term "cancer survivor" was coined by Fitzhugh Mullan, a physician and cancer patient, to describe his own experience dealing with cancer survivorship [1]. Recent advances in cancer treatment have successfully prolonged cancer survival. Recently, the Surveillance, Epidemiology, and End Results (SEER) program has reported that the 5-year survival rate of breast cancer treatment is almost 90\% [2]. Whilst the definition of being cured itself is still controversial [3], the medical and psychosocial problems encountered by cancer survivors are well established [4]. For example, the combination of surgery, chemotherapy, and/or radiotherapy often lead to fatigue and decrease in patients' quality of life [5].

\footnotetext{
* Corresponding author: Tjing Yung Loo

Keenlink Dental Clinic \& Essence Medical Laboratory, Hong Kong.

Copyright (C) 2021 Author(s) retain the copyright of this article. This article is published under the terms of the Creative Commons Attribution Liscense 4.0.
} 
Numerous musculoskeletal problems may arise after medical treatment. In particular, breast cancer survivors who had received radical mastectomy or modified mastectomy might have impaired muscular strength and shoulder mobility as they often underwent a more extensive lymph node resection [6]. Survivors often report fatigue, pain, and lymphedema [7-9]. Although the American Society of Clinical Oncology (ASCO) and the American Cancer Society (ACS) joint guidelines on breast cancer survivorship highlight the importance of exercise [4], the aforementioned factors restrict their ability to perform conventional exercises. Hence, breast cancer survivors might have difficulties to reap the benefits of exercise, such as improved natural killer cell cytotoxic activity and reduced cancer recurrence [10-11].

In this study, we investigated the benefits of practicing Tai Chi Chuan (TCC) on post-treatment breast cancer survivors. TCC is a traditional Chinese exercise emphasizing on fluid and slow rhythm. The movements of TCC are gentle and smooth, guided by natural breathing pattern. Thus, TCC practice may be an option of exercise in rehabilitation program, thus avoiding strenuous exercises on the already fatigued post-treatment patients

The benefits of TCC on improving muscle strength and functional wellbeing have been reported in previous studies [1215]. Our previous studies have also demonstrated that the white blood cells, red blood cells, and hemoglobin of breast cancer survivors had recovered faster in those who practiced TCC 45 minutes a day, six times a week for 1 year [16-17]. However, the value of TCC practice to improve immune system and decrease pro-metastasis markers in early posttreatment breast cancer survivors is yet to be explored.

Although Natural Killer (NK) cells comprise only 5 to $20 \%$ of peripheral blood lymphocytes, they are known to have a vital role in cancer immunosurveillance [16]. Waldhauer and Steinle have previously reported on the major activating NK receptors such as NCR NKp30, NKp44 and NKp46, and the CTLR NKG2D [18]. Among those, NKG2D was chosen as our outcome measure since Mamessier and colleagues reported on its downregulation after direct or indirect contact with primary breast tumor cells [19]. In this study, we also investigated whether TCC practice could reduce the prometastasis markers such as P-selectin, which is being implicated to shield tumor cells from NK-mediated lysis [20-21]; and vascular endothelial growth factor (VEGF), a protein that is crucial for tumor metastasis through the VEGF-VEGFR1 signaling pathway [22].

\section{Material and methods}

\subsection{Participants}

130 post-treatment breast cancer survivors were recruited and randomized 1:1 into TCC group and wait-list (control) group. The TCC group practiced a 60-minutes session once per week, for a total of 52 weeks. 115 forms of Yang-style TCC were taught by a qualified Tai Chi master. The wait-list (control) group received same intervention of TCC after TCC group completing 52 weeks exercise. This study was approved by the ethics committee of The Hong Kong Polytechnic University. Informed consent was obtained from all individual participants included in the study

Eligible participants were defined as breast cancer survivors aged 30 to 55 years who had received conventional cancer treatment(s), including single/two sides radical or modified mastectomy, chemotherapy and/or radiotherapy within 6 months, and without any systemic diseases. Patients were excluded if chemotherapy had been completed for over 6 months. In addition, 32 healthy volunteers were also recruited from the community for the baseline comparison of NKG2D, VEGF, and P-selectin expression with the survivors. After consent, the blood samples were collected by direct venipuncture from the arm vein of the participants. A total of $6 \mathrm{ml}$ was collected into the EDTA tubes.

\subsection{Expression of NKG2D, P-selectin, VEGF}

The samples were collected to perform a complete blood count (CBC) using the Sysmex Xs-1000i automation machine (Sysmex, Japan). In order to separate plasma from the blood samples, a further centrifugation step was performed for 10 minutes at 1,500 rpm. The plasma was then isolated for enzyme linked immunosorbent assay (ELISA). The human NKG2D protein sandwich ELISA kits (Cusabio, United States) have been exploited throughout the assay. A sandwich ELISA requires two-step staining, also known as indirect immunolabelling. The expressions of VEGF and P-selectin were measured with VEGF ELISA kit (Peviva, Sweden) and P-selectin ELISA kit (Diaclone, France), respectively.

\subsection{Lymphocyte's isolation and cell culture}

Peripheral blood mononuclear cells were purified out by the Ficoll-Paque (Amersham Biosciences, Sweden). The isolated lymphocytes were cultured into 96-well plates for 72 hours with the addition of $20 \mathrm{ug} / \mathrm{ml}$ PHA, a carbohydratebinding protein that stimulates the cell division of lymphocytes. Freshly harvested, the PHA-stimulated lymphocytes 
were incubated with 10ul Cell Proliferation Reagent WST-1 (Roche, Germany). The absorbance was read at $450 \mathrm{~nm}$ on a spectrophotometer; the increased absorbance was directly proportional to cell activity.

\subsection{ATP bioluminescence assay}

The lymphocytes were plated onto a $25 \mathrm{~cm}^{2}$ cell culture flask at the initial concentration of $1 \times 10^{6}$ cells $/ \mathrm{ml}$. PHA was added and the incubation time was $72 \mathrm{~h}$. Freshly harvested, the metabolic rate of lymphocytes was measured by ATP bioluminescence assay kit HS II (Roche, Germany). The procedure was reproduced from our previous experiment [23]. The lymphocytes were transferred to microcentrifuge tubes and $0.5 \mathrm{ml}$ cell lysis reagent was added. Afterwards, to prepare the luciferase reagent of the ATP bioluminescence assay, $10 \mathrm{ml}$ dilution buffer was added to the bottle of luciferase reagent as supplied by the kit. Cell lysis was accelerated by a sonicator (Sonics \& Materials, Inc. Danbury, CT. USA) at a pulse of 30 per minute with $20 \%$ amplitude, for 1 min. There was a 10 -sec binding period preceded by amalgamation of $50 \mu \mathrm{l}$ of samples and $50 \mu \mathrm{l}$ luciferase reagent. The absorbance value of the samples was read by TD20/20 luminometer (Turner Designs, CA. USA) at $420 \mathrm{~nm}$ wavelength. As an indirect measurement of ATP, the assay kit provides a standard ATP for conversion of the samples' optical densities to bioluminescence (rlu). A standard ATP curve was prepared with material supplied by the kit.

\subsection{Colony formation of T-lymphocytes transformation}

The culture of the lymphocytes was similar to the condition for ATP bioluminescence assay. Freshly harvested, lymphocytes were plated to $150 \times 20 \mathrm{~mm}$ tissue culture dish. Subsequently, images were taken with an inverted microscope (Motic, Canada). The area of lymphocyte cluster or colony was measured using an ocular micrometer.

\subsection{Statistical Analysis}

All the data analyses were conducted using SPSS 26.0 (version 26, IBM Corp., Armonk, NY, USA) and expressed as mean \pm standard deviation (SD). Repeated measure analysis of variance (ANOVA) was performed to compare the differences between TCC and wait-list groups at baseline and week-52. Comparison of the mean area of lymphocyte clusters or colonies at week-52 was performed using unpaired t-test. $P<0.05$ was considered statistically significant.

\section{Results}

\subsection{Patient characteristics}

The mean age of patients in the TCC group was 41.2 years (age range, 28 to 54 years) and the mean age in wait-list group was 43.5 years (age range, 29 to 68 years). For the type of surgery, both group had similar proportions of patients who underwent modified radical mastectomy (MRM). Six patients in TCC group and 7 patients in wait-list group did not go through MRM. Healthy volunteers were recruited from the community and their mean age was 31.6 years (age range, 18 to 42 years).

\subsection{NKG2D, VEGF, and P-selectin}

Table 1 Expression of NKG2D protein, P-selectin, and VEGF in plasma at baseline and 52 weeks

\begin{tabular}{|l|c|c|c|c|c|}
\hline \multicolumn{1}{|c|}{ Parameters } & $\begin{array}{c}\text { TCC } \\
\text { (Baseline) } \\
\text { Mean } \pm \text { SD }\end{array}$ & $\begin{array}{c}\text { Wait-list } \\
\text { (Baseline) } \\
\text { Mean } \pm \text { SD }\end{array}$ & $\begin{array}{c}\text { TCC } \\
\text { (52 weeks) } \\
\text { Mean } \pm \text { SD }\end{array}$ & $\begin{array}{c}\text { Wait-list } \\
\text { (52 weeks) } \\
\text { Mean } \pm \text { SD }\end{array}$ & $\begin{array}{c}\text { Healthy } \\
\text { volunteers } \\
\text { Mean } \pm \text { SD }\end{array}$ \\
\hline NKG2D. $(\mathrm{ng} / \mathrm{mL})$ & $0.08 \pm 0.01$ & $0.08 \pm 0.01$ & $* 0.19 \pm 0.02$ & $0.16 \pm 0.02$ & $0.22 \pm 0.02$ \\
\hline VEGF. $(\mathrm{pg} / \mathrm{mL})$ & $117.9 \pm 46.6$ & $121.4 \pm 41.4$ & $* * 44.4 \pm 10.2$ & $50.6 \pm 14.1$ & $43.4 \pm 13.6$ \\
\hline P-selectin $(\mathrm{ng} / \mathrm{mL})$ & $84.47 \pm 13.1$ & $85.83 \pm 11.1$ & $43.7 \pm 9.6$ & $46.28 \pm 7.9$ & $36.5 \pm 4.6$ \\
\hline
\end{tabular}

$N K G 2 D$, natural-killer group 2 member D; $S D$, standard deviation; $V E G F$, vascular endothelial growth factor.

* Significant difference compared to wait-list group at 52 weeks $(p=0.001)$; ${ }^{* *}$ significant difference compared to wait-list group at 52 weeks $(p=0.005)$

The values for NKG2D, VEGF, and P-selectin for healthy volunteers (at baseline), TCC and wait-list group (baseline and 52 weeks) can be seen in Table 1. Compared against healthy volunteers, both TCC and wait-list groups had lower NKG2D values, and higher P-selectin and VEGF values. At 52 weeks, TCC group had a significantly higher NKG2D value ( $p=0.001)$ 
and a significantly lower VEGF value ( $p=0.005$ ) compared to wait-list group, however, there was a small, non-significant change for P-selectin values between both breast cancer survivor groups.

\subsection{Lymphocyte proliferation and T-lymphocyte transformation}

Based on CBC, the white blood cells (WBC), red blood cells (RBC), and hemoglobin ( $\mathrm{Hb}$ ) of both groups at baseline and 52 weeks are presented in Table 2. WBC was significantly higher in TCC group as compared to wait-list group at 52 weeks $(p=0.001)$. After $72 \mathrm{~h}$ incubation, the TCC group had a significant increase in lymphocyte proliferation $(p=0.001)$ as compared to wait-list group (Table 3). As described by Lange, the formation of lymphocyte cluster or colonies was defined as aggregates of cells from 3 to hundreds [24]. The area of the lymphocyte clusters or colonies was visibly larger in TCC group, as seen in Fig. 1. At 52 weeks, the mean area was significantly different between both groups $(p=0.001)$. It was $7.11 \pm 1.38 \mathrm{~mm}^{2}$ for wait-list group and $15 \pm 3.2 \mathrm{~mm}^{2}$ for TCC group.

Table 2 Selected parameters from CBC at baseline and 52 weeks

\begin{tabular}{|l|c|c|c|c|c|}
\hline \multicolumn{1}{|c|}{ Parameters } & $\begin{array}{c}\text { TCC } \\
\text { (Baseline) } \\
\text { Mean } \pm \text { SD }\end{array}$ & $\begin{array}{c}\text { Wait-list } \\
\text { (Baseline) } \\
\text { Mean } \pm \text { SD }\end{array}$ & $\begin{array}{c}\text { TCC } \\
\text { (52 weeks) } \\
\text { Mean } \pm \text { SD }\end{array}$ & $\begin{array}{c}\text { Wait-list } \\
\mathbf{5 2} \text { weeks) } \\
\text { Mean } \pm \text { SD }\end{array}$ & $\begin{array}{c}\text { Normal } \\
\text { Range }\end{array}$ \\
\hline WBC. $\left(\mathrm{x} 10^{9} / \mathrm{L}\right)$ & $3.32 \pm 0.83$ & $3.50 \pm 0.88$ & $* 5.22 \pm 0.78$ & $4.12 \pm 0.43$ & $4-11$ \\
\hline RBC. $\left(\mathrm{x} 10^{12} / \mathrm{L}\right)$ & $3.20 \pm 0.65$ & $3.05 \pm 0.60$ & $5.43 \pm 0.92$ & $5.05 \pm 0.99$ & $3.8-5.8$ \\
\hline $\mathrm{Hb}$ (g/dL) & $9.05 \pm 1.57$ & $9.50 \pm 1.66$ & $13.11 \pm 1.35$ & $13.22 \pm 1.89$ & $11.5-16.5$ \\
\hline
\end{tabular}

* Significant difference compared to wait-list group at 52 weeks $(p=0.001)$

Table 3 Lymphocyte proliferation after $72 \mathrm{~h}$ incubation

\begin{tabular}{|l|c|c|c|c|}
\hline & \multicolumn{2}{|c|}{$\begin{array}{c}\text { Cell proliferation in OD } \\
\text { Mean } \pm \text { SD }\end{array}$} & $\begin{array}{c}\text { Metabolic activities of lymphocytes in ATP(rlu) } \\
\text { Mean } \pm \text { SD }\end{array}$ \\
\hline Groups & Baseline & After $72 \mathrm{~h}$ & Baseline & After $72 \mathrm{~h}$ \\
\hline TCC & $0.77 \pm 0.25$ & $* 1.95 \pm 0.6$ & $1.23 \pm 0.31$ & $* 4.42 \pm 0.78$ \\
\hline Wait-list & $0.77 \pm 0.28$ & $1.32 \pm 0.37$ & $1.25 \pm 0.32$ & $2.08 \pm 0.28$ \\
\hline
\end{tabular}

The proliferative rate and metabolic activity were measured in OD by WST-1 cell proliferation reagent and ATP bioluminescence assay $O D$, optical density; $S D$, standard deviation

* Significant difference compared to wait-list group at $72 \mathrm{~h}(p=0.001)$

\subsection{Figure legend}

Figure 1. Colony formation of T-lymphocytes transformation observed under an inverted microscope. (A) wait-list group at baseline; (B) wait-list group after 72h incubation with PHA; (C) TCC group at baseline; (D) TCC group after 72h incubation with PHA. PHA, phytohaemaglutinin; TCC, Tai Chi Chuan. 


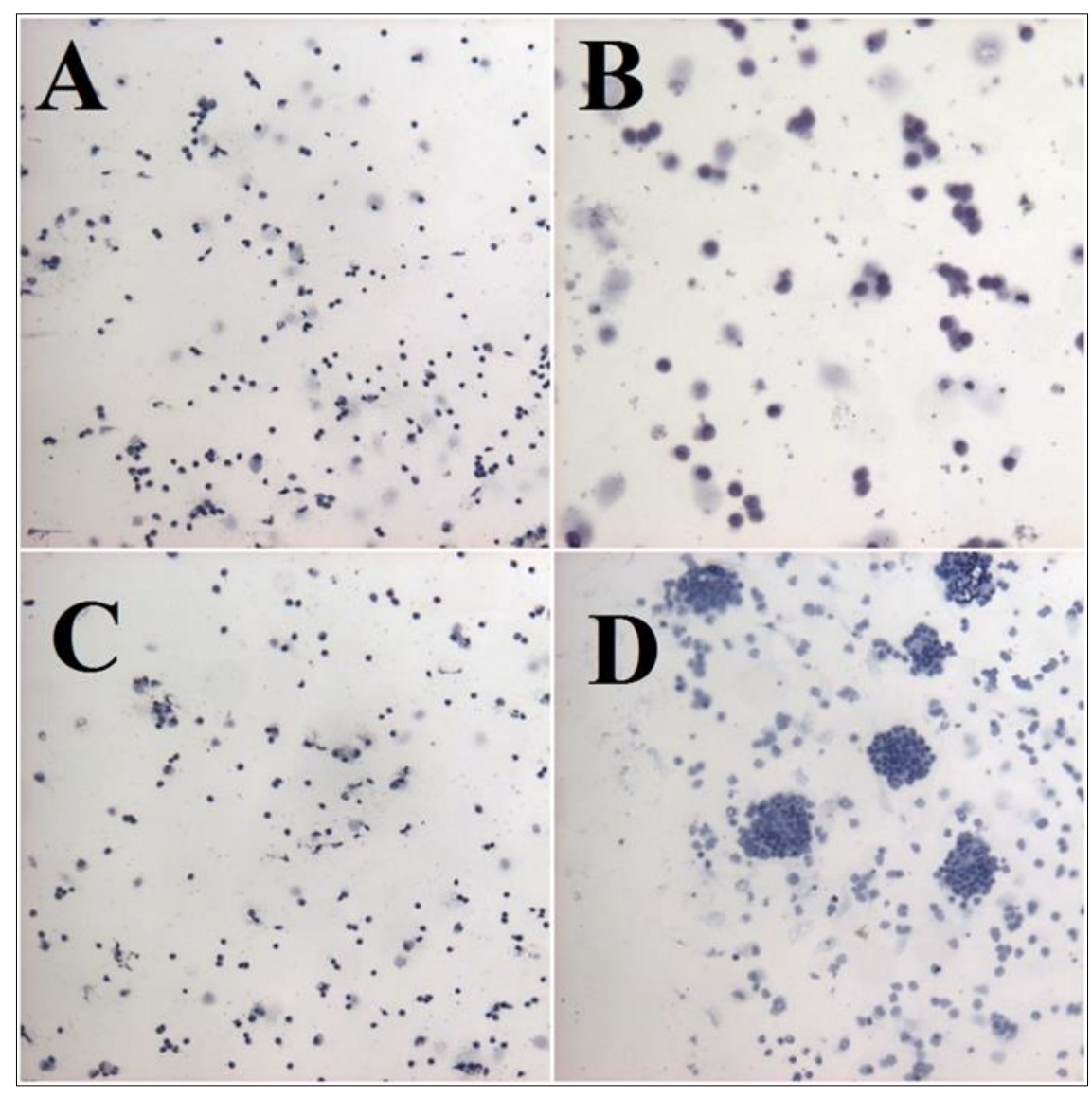

Figure 1 Colony formation of T-lymphocytes transformation observed under an inverted microscope. (A) wait-list group at baseline; (B) wait-list group after 72h incubation with PHA; (C) TCC group at baseline; (D) TCC group after $72 \mathrm{~h}$ incubation with PHA.

PHA, phytohaemaglutinin; TCC, Tai Chi Chuan.

\section{Discussion}

The present study has demonstrated that breast cancer survivors in the TCC group had improved WBC value, increased lymphocyte proliferation and colony formation, improved expression of NKG2D in plasma, and reduced VEGF in plasma. Nonetheless, our results failed to demonstrate significant reductions in P-selectin by practicing TCC. In this study, the blood samples of our healthy volunteers also underwent similar sample preparation as the TCC and wait-list's blood samples. As various reference values were reported depending on the procedure, sample type, and assay manufacturer [26-27], the values obtained from healthy volunteers act only as a reference point in our study. We note that the mean age of our healthy volunteers was younger, thus we did not have the intention to directly compare the values to our main study groups.

The improvement of NKG2D and reduction of VEGF expressions in plasma - closer to the value of healthy volunteers, may present as a valuable benefit of practicing TCC, as these markers contributed to the suppression of tumor and the metastases. NKG2D was previously reported to be downregulated after contact with tumor cells [19], whereas elevated levels of VEGF are also related to a higher chance of recurrence of cancer or even death [27]. Breast cancer is an angiogenesis-dependent tumor, largely relying on VEGF signaling for the angiogenic response [27-29].

Meanwhile, the lymphocyte proliferative response to PHA has also been known to be impaired in cancer patients [30]. The diminished cellular immune function in our breast cancer survivor group, however, appears to be improved significantly on the patients who practiced TCC. As Goon et al demonstrated in their study on healthy adults aged over 45, TCC participants for 7 years had elevated lymphocyte proliferation response to PHA, possibly helping to restore decreased immune response related to the replicative senescence associated with aging [31]. Hence, the benefit of improved immune response appears to be extended to our population of early post-treatment breast cancer survivors as well. 
As with all studies, limitations exist in our study. First, we consider blinding to be impractical and difficult to implement for our study, however, we note that the outcome assessor in our study was blinded. Second, we are also aware of possible confounding factors in our samples, for example, we were not able to screen and categorize samples based on their previous chemotherapy regime. As described by Mills [32], post-chemotherapy soluble P-selectin values may vary depending on the chemotherapy regime; reportedly highest in women who had received epirubicin, cyclophosphamide, and 5-fluorouracil. Hence, this might explain why our results did not show significant improvement in P-selectin by practicing TCC. Thirdly, we did not compare a control group undergoing conventional exercise regime. Whether the beneficial effect is due to TCC practice or exercise per se requires further investigation.

In conclusion, as an alternative for conventional exercises, post-treatment breast cancer survivors may opt for TCC to be implemented in their rehabilitation program. The practice of TCC could help stimulate tumor immunosurveillance via NKG2D and activate immune response. VEGF, a marker playing an important role in breast cancer and its metastases, was also reduced in those who practiced TCC.

\section{Conclusion}

Practicing Tai Chi Chuan would boost immune system and relieve side effects from surgery, chemotherapy, and/or radiotherapy in breast cancer survivors, eventually, improving their quality of life.

\section{Compliance with ethical standards}

\section{Acknowledgments}

We would like to thank Dr William Tsang of Department of Rehabilitation Sciences, The Hong Kong Polytechnic University for recruiting breast cancer survivors and assisting management and supervision of TCC project.

\section{Disclosure of conflict of interest}

The authors declare no potential conflicts of interest.

\section{Statement of informed consent}

Informed consent was obtained from all individual participants included in the study.

\section{References}

[1] Mullan F. Seasons of survival: reflections of a physician with cancer. N Engl J Med. 1985; 313(4): 270-3.

[2] Howlader N, Noone AM, Krapcho M, Garshell J, Miller D, Altekruse SF, et al [eds]. SEER Cancer Statistics Review, 1975-2012, National Cancer Institute. Bethesda, MD. April 2015.

[3] Surbone A, Tralongo P. Categorization of Cancer Survivors: Why We Need It. J Clin Oncol. 2016; 34(28): 3372-4.

[4] Runowicz CD, Leach CR, Henry NL, Henry KS, Mackey HT, Cowens-Alvarado RL, et al. American Cancer Society/American Society of Clinical Oncology Breast Cancer Survivorship Care Guideline. CA Cancer J Clin. 2016; 66(1): 43-73.

[5] Fong DY, Ho JW, Hui BP, Lee AM, Macfarlane DJ, Leung SS, et al. Physical activity for cancer survivors: metaanalysis of randomised controlled trials. BMJ. 2012; 344: e70.

[6] Thomas-Maclean RL, Hack T, Kwan W, Towers A, Miedema B, Tilley A. Arm morbidity and disability after breast cancer: new directions for care. Oncol Nurs Forum. 2008; 35(1): 65-71.

[7] Young KE, White CA. The prevalence and moderators of fatigue in people who have been successfully treated for cancer. J Psychosom Res. 2006; 60(1): 29-38.

[8] Sekse RJ, Raaheim M, Blaaka G, Gjengedal E. Life beyond cancer: women's experiences 5 years after treatment for gynaecological cancer. Scand J Caring Sci. 2010;24(4): 799-807.

[9] Jereczek-Fossa BA, Marsiglia HR, Orecchia R. Radiotherapy-related fatigue. Crit Rev Oncol Hematol. 2002; 41(3): 317-25. 
[10] Fairey AS, Courneya KS, Field CJ, Bell GJ, Jones LW, Mackey JR. Randomized controlled trial of exercise and blood immune function in postmenopausal breast cancer survivors. J Appl Physiol [1985]. Apr 2005; 98(4): $1534-40$.

[11] Dieli-Conwright CM, Lee K, Kiwata JL. Reducing the Risk of Breast Cancer Recurrence: an Evaluation of the Effects and Mechanisms of Diet and Exercise. Curr Breast Cancer Rep. 2016; 8(3): 139-150.

[12] Zhou M, Peng N, Dai Q, Li HW, Shi RG, Huang W. Effect of Tai Chi on muscle strength of the lower extremities in the elderly. Chin J Integr Med. 2016; 22(11): 861-866.

[13] Sun W, Zhang C, Song Q, Li W, Cong Y, Chang S, et al. Effect of 1-year regular Tai Chi on neuromuscular reaction in elderly women: a randomized controlled study. Res Sports Med. 2016; 24(2): 145-56.

[14] Fong SS, Ng SS, Luk WS, Chung JW, Chung LM, Tsang WW, et al. Shoulder Mobility, Muscular Strength, and Quality of Life in Breast Cancer Survivors with and without Tai Chi Qigong Training. Evid Based Complement Alternat Med. 2013; 787169.

[15] Mustian KM, Palesh OG, Flecksteiner SA. Tai Chi Chuan for breast cancer survivors. Med Sport Sci. 2008; 52: 20917.

[16] Tsang WWN, et al. Effect of Tai Chi Chuan on serotonin and cortisol for monitoring stress and quality of life in post-treatment breast cancer patients. Chin J Breast Dis [Electronic Edition]. 2014; 4: 009.

[17] Loo WTY, et al. Tai Chi Chuan improves cytokine expression levels and quality of life in post chemotherapy breast cancer patients. Int J Biol Markers. 2016.

[18] Waldhauer I, Steinle A. NK cells and cancer immunosurveillance. Oncogene. 2008; 27: 5932-5943.

[19] Mamessier E, Sylvain A, Bertucci F, Castellano R, Finetti P, Houvenaeghel G, et al. Human breast tumor cells induce self-tolerance mechanisms to avoid NKG2D-mediated and DNAM-mediated NK cell recognition. Cancer Res. 2011; 71(21): 6621-32.

[20] Placke T, Salih HR, Kopp HG. GITR ligand provided by thrombopoietic cells inhibits NK cell antitumor activity. J Immunol. 2012; 189(1): 154-60.

[21] Coupland LA, Chong BH, Parish CR. Platelets and P-selectin control tumor cell metastasis in an organ-specific manner and independently of NK cells. Cancer Res. 2012; 72(18): 4662-71.

[22] Liu W, Xu J, Wang M, Wang Q, Bi Y, Han M. Tumor-derived vascular endothelial growth factor [VEGF]-a facilitates tumor metastasis through the VEGF-VEGFR1 signaling pathway. Int J Oncol. 2011; 39(5): 1213-20.

[23] Loo WT, Jin LJ, Chow LW, Cheung MN, Wang M. Rhodiola algida improves chemotherapy-induced oral mucositis in breast cancer patients. Expert Opin Investig Drugs. 2010; 19 Suppl 1: S91-100.

[24] Lange BJ. Growth of human leukemia cells in vitro. In: Baserga R, editor, Cell growth and division: a practical approach. Oxford: Oxford University Press. 1989; 61-80.

[25] Thom J, Gilmore G, Yi Q, Hankey GJ, Eikelboom JW. Measurement of soluble P-selectin and soluble CD40 ligand in serum and plasma. J Thromb Haemost. 2004; 2: 2067-9.

[26] Kut C, Mac Gabhann F, Popel AS. Where is VEGF in the body? A meta-analysis of VEGF distribution in cancer. Br J Cancer. 2007; 97(7): 978-85.

[27] Gasparini G. Prognostic value of vascular endothelial growth factor in breast cancer. Oncologist. 2000; 5 Suppl 1: 37-44.

[28] Schneider BP, Miller KD. Angiogenesis of breast cancer. J Clin Oncol. 2005; 23(8): 1782-90.

[29] Fox SB, Generali DG, Harris AL. Breast tumour angiogenesis. Breast Cancer Res. 2007; 9(6): 216.

[30] Melichar B, Jandik P, Krejsek J, Solichova D, Drahosova M, Skopec F, et al. Mitogen-induced lymphocyte proliferation and systemic immune activation in cancer patients. Tumori. 1996; 82(3): 218-20.

[31] Goon JA, Noor Aini AH, Musalmah M, Yasmin Anum MY, Wan Ngah WZ. Long term Tai Chi exercise reduced DNA damage and increased lymphocyte apoptosis and proliferation in older adults. Med J Malaysia. 2008; 63(4): 31924.

[32] Mills PJ, Ancoli-Israel S, Parker B, Natarajan L, Hong S, Jain S, et al. Predictors of inflammation in response to anthracycline-based chemotherapy for breast cancer. Brain Behav Immun. 2008; 22(1): 98-104. 